\title{
PRESENCIA DE HIENA MANCHADA EN LOS DEPÓSITOS BASALES (PLEISTOCENO SUPERIOR FINAL) DEL YACIMIENTO ARQUEOLÓGICO DE LA CUEVA DE NERJA (MÁLAGA, ESPAÑ̃)
}

Trabajo presentado a las XVIII Jornadas de Paleontología y II Congreso Ibérico de Paleontología Universidad de Salamanca

Salamanca, 24-29 de Septiembre de 2002

\author{
Alfonso ARRIBAS HERRERA ${ }^{1}$, J. Emilio AURA \\ TORTOSA ${ }^{2}$, JOSé S. CARRIÓN ${ }^{3}$, Jesús F. JORDÁ \\ PARDO 4 y Manuel PÉREZ RIPOLL ${ }^{2}$
}

\footnotetext{
${ }^{1}$ Museo Geominero. Instituto Geológico y Minero de España. Ríos Rosas, 23. 28003 Madrid. a.arribas@igme.es

${ }^{2}$ Departamento de Prehistòria i Arqueologia. Universitat de València. Blasco Ibañez, 28. 46001 València. emilio.aura@uv.es

${ }^{3}$ Departamento de Biología Vegetal. Facultad de Biología. Universidad de Murcia.30100 Murcia. carrion@um.es

${ }^{4}$ Departamento de Prehistoria e Historia Antigua. Facultad de Geografía e Historia. UNED. Ciudad Universitaria. C/ Senda del Rey, 7. 28040 Madrid. jjorda@geo.uned.es
}

Arribas Herrera, A., Aura Tortosa, J.E., Carrión, J.S., Jordá Pardo, J.F. y Pérez Ripoll, M. 2004. Presencia de hiena manchada en los depósitos basales (Pleistoceno superior final) del yacimiento arqueológico de la Cueva de Nerja (Málaga, España). [Fossil spotted hyaena presence at the basal deposits (Upper Pleistocene) of the archaeological site of Nerja's Cave (Málaga, Spain).] Revista Española de Paleontología, 19 (1), 109-121. ISSN 0213-6937.

\begin{abstract}
In the final stage of the Upper Pleistocene and most part of the Holocene, a strong stratigraphic series was placed on the access (Torca, Mina and Vestíbulo Halls) of Nerja's Cave (Maro, Nerja, province of Málaga, South of Spain). From basal level of Vestibulo Hall (NV13, square C-4 y B-8), $c a$. 25,000 and $c a$. 20,000 years BP (Upper Pleistocene), proceeded four fragmets of white coprolites adscribed to fossil spotted hyaena (Crocuta crocuta spelaea).
\end{abstract}

Key words: Palaeobiological record, fossil spotted hyaena, Karst, Pleistocene, Gravetien, Nerja's Cave, Málaga, South Spain.

\section{RESUMEN}

La Cueva de Nerja (Maro, témino municipal de Nerja, provincia de Málaga, S de España) contiene un importante yacimiento arqueológico en la zona de su antigua entrada (salas de la Torca, de la Mina y del Vestíbulo) cuya cronología se encuentra comprendida entre $c a .25 .000$ y $c a .3 .000$ años BP, secuencia que cubre el Pleistoceno superior final y gran parte del Holoceno. En el nivel basal de la Sala del Vestíbulo (NV13, cuadros C-4 y B-8), acotado en una horquilla cronológica comprendida entre $c a .25 .000$ y $c a .20 .000$ años BP, se han recuperado cuatro fragmentos de coprolito de color blanco atribuibles la hiena manchada de las cavernas (Crocuta crocuta spelaea).

Palabras clave: Registro paleobiológico, hiena manchada de las cavernas, Karst, Pleistoceno, Gravetiense, Cueva de Nerja, Málaga, Sur de España. 


\section{INTRODUCCIÓN}

La Cueva de Nerja (Maro, témino municipal de Nerja, provincia de Málaga, S de España) (Fig. 1) contiene un importante yacimiento arqueológico en la zona de su antigua entrada (salas de la Torca, de la Mina y del Vestíbulo) (Fig. 2b) cuya cronología se encuentra comprendida entre $c a .25 .000$ y $c a .3 .000$ años BP, secuencia que cubre el Pleistoceno superior final y gran parte del Holoceno (Aura et al., 1998, 2002a).

El registro paleobiológico de los depósitos finipleistocenos y holocenos de la Cueva de Nerja cuyas características se han dado a conocer de forma sintética en una reciente publicación (Jorda Pardo et al., 2003) comprende una treintena de taxones vegetales (Badal, 1990, 1991, 1996, 1998), casi un centenar de especies de numerosas clases de invertebrados (Jordá Pardo, 1981, 1982, 1983, 1985, 1986b; Serrano et al., 1995) y un centenar y medio de taxones de vertebrados entre peces, reptiles, aves y mamíferos (Boessneck y Driesch, 1980; Eastham, 1986; Jiménez Fuentes, 1986; Pérez Ripoll, 1986, 1997; Rodrigo, 1991; Hernández, 1995; Morales y Martín, 1995; Roselló et al., 1995; Guillém, 1997). Cuantitativamente y considerando únicamente los materiales procedentes de las excavaciones del Dr. Jordá Cerdá, los restos antracológicos estudiados hasta el momento superan los 13.000, mientras que los de invertebrados alcanzan los 200.000 y los de vertebrados rondan los 30.000 (teniendo en cuenta que los peces han sido estudiados a partir de una muestra).

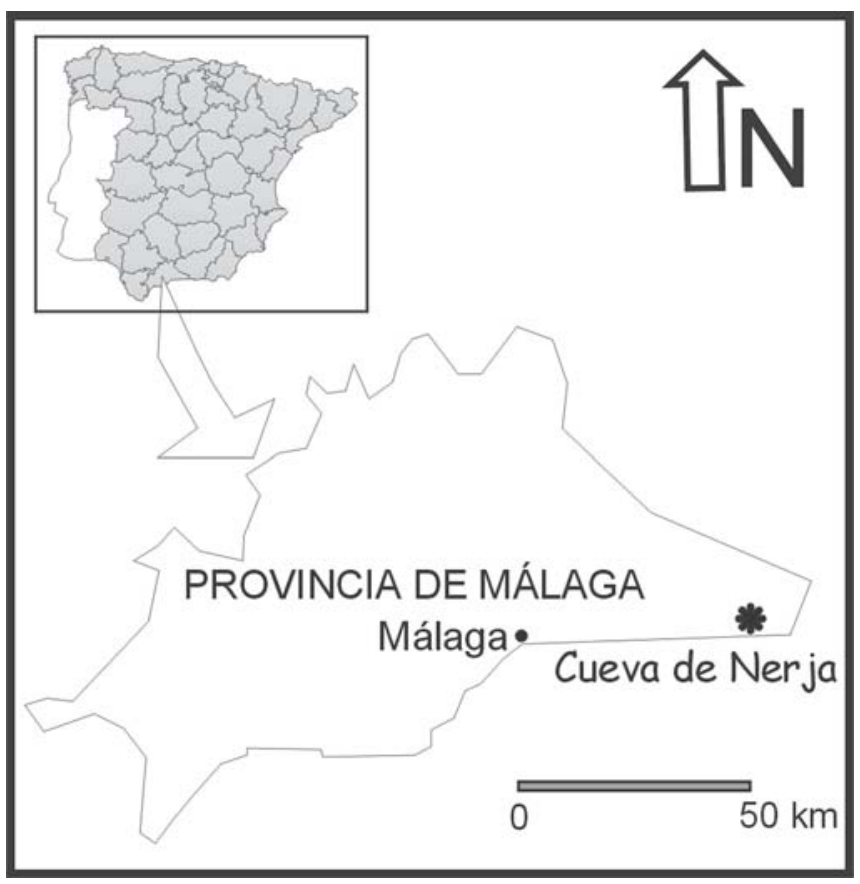

Figura 1. Situación geográfica de la Cueva de Nerja (Málaga, España).

Geographic setting of Nerja's Cave (Málaga, Spain).
En el nivel basal del Vestíbulo (NV13, cuadros C-4 y B-8) se han recuperado cuatro fragmentos de coprolitos de color blanco atribuibles a un gran carnívoro por sus dimensiones centimétricas (actualmente depositados para su estudio en el Dpto. de Prehistoria y Arqueología de la Universidad de Valencia). En este paquete sedimentario se han recuperado además diferentes restos paleontológicos (Equus sp., Bos sp., Cervus elaphus Linneo, 1758 y Capra pyrenaica Schinz, 1838) y algunos útiles líticos, cuya densidad se incrementa hacia el techo del nivel.

El objeto del presente trabajo es dar a conocer los citados fragmentos de coprolitos que incrementan el registro paleontológico de los depósitos situados en la zona de la entrada de la Cueva de Nerja (salas del Vestíbulo, de la Mina y de la Torca), de edad Pleistoceno superior final Holoceno, cuyos componentes tecnológicos, vegetales y animales son bien conocidos (Jordá Pardo et al., 1990, 2003; Aura et al., 1998, 2000, 2002a).

\section{CONTEXTO ESTRATIGRÁFICO}

Geológicamente, la Cueva de Nerja se encuentra situada en el borde suroccidental del macizo de Almijara, unidad de relieve que se extiende al NE de la falla de Frigiliana y se desarrolla sobre los mármoles dolomíticos fuertemente recristalizados de edad triásica. Estos materiales constituyen la unidad superior del Manto de La Herradura (Complejo Alpujárride, Cordillera Bética) y configuran la Sierra Almijara en cuya vertiente meridional, al pie de la Ladera del Águila, se desarrolla la cavidad. En el exterior, apenas unos metros por debajo de la entrada a la cueva se extiende el abanico aluvial de Maro, de edad Pleistoceno (Jorda Pardo, 1992), que con una ligera pendiente llega a la costa donde se ve cortado de manera abrupta dando lugar a un escarpado acantilado. Este abanico se encuentra disectado por el barranco de Maro que origina un profundo cañón en cuya desembocadura existe una pequeña playa.

Los depósitos detríticos y químicos que colmatan parcialmente la zona de la antigua entrada de la Cueva de Nerja (salas de la Torca, de la Mina y del Vestíbulo) se disponen en el Vestíbulo por encima de un grueso espeleotema y constituyen el yacimiento arqueológico sobre el que se han centrado nuestras investigaciones (Aura et al., 1998, 2002a). Su cronología se encuentra comprendida entre las fechas de radiocarbono UBAR-340 24.300 $\pm 1.400 \mathrm{BP}$ procedente de la base de la secuencia del Vestíbulo (Nivel N.V.13)(Aura et al., 2001) y GAK-8960 2.860 \pm 220 BP obtenida en los depósitos de la Torca (NT-79/ 2)(Pellicer y Acosta, 1997), cubriendo el Pleistoceno superior final y gran parte del Holoceno y los estadios isotópicos 2 y 1 (Shackleton y Opdyke, 1973). A partir de la correlación de las secuencias litoestratigráficas, obtenidas en las excavaciones arqueológicas sistemáticas reali- 

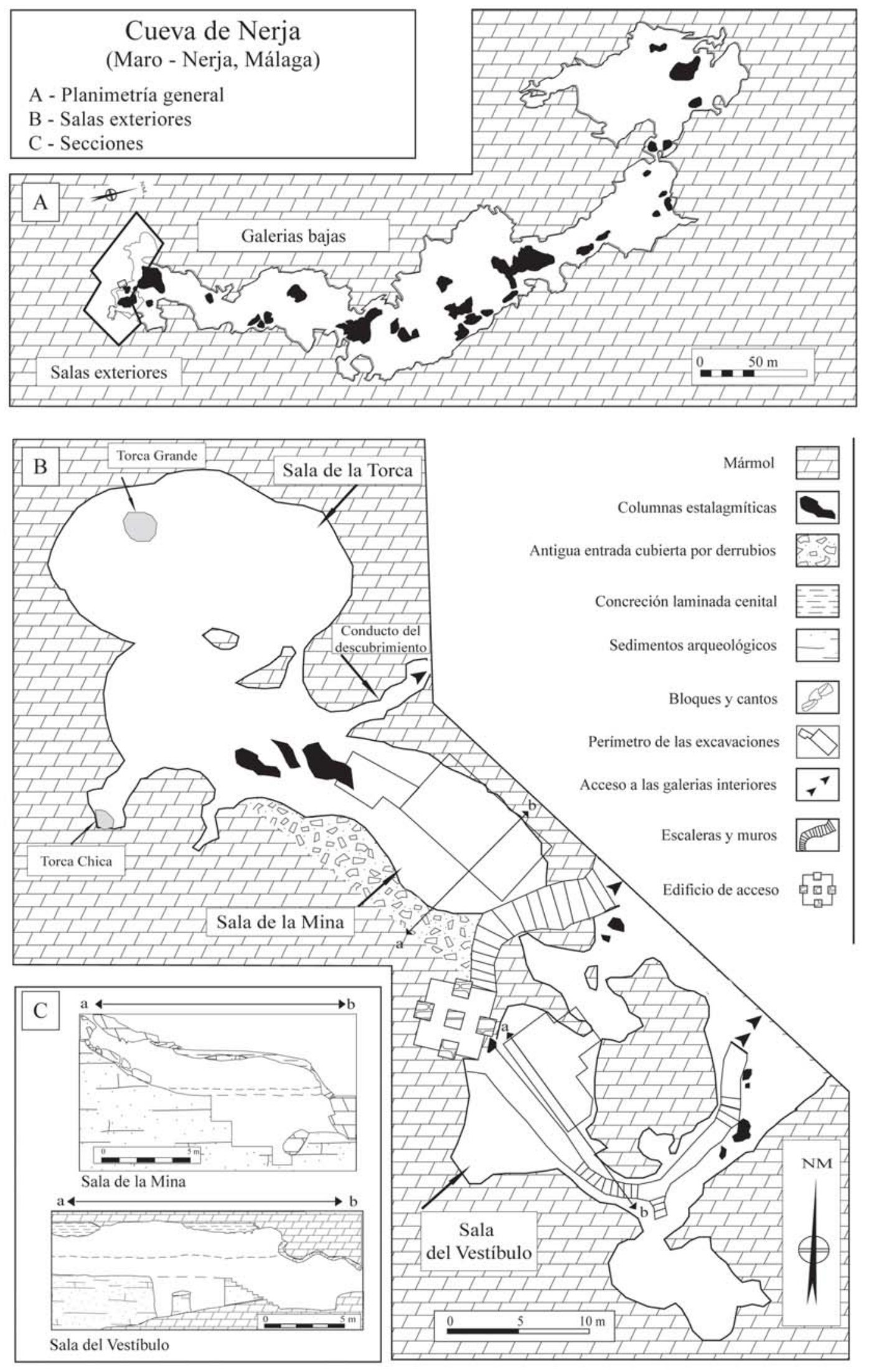

Figura 2. Antigua entrada de la Cueva de Nerja, indicando las zonas con registros estudiados. Ancient access of Nerja's Cave, showing the areas with studied records. 
zadas en los depósitos de las salas de la Mina y del Vestíbulo dirigidas por el profesor Dr. Jordá Cerdá (subvencionadas entre 1979 y 1987 por el Patronato de la Cueva de Nerja y autorizadas por la Junta de Andalucía), hemos obtenido la secuencia cronoestratigráfica sintética del yacimiento (Jordá Pardo, 1986a; Jordá Pardo et al., 1990; Aura Tortosa et al., 1998, 2000, 2002a). En esa secuencia, hasta la fecha, distinguimos doce etapas de sedimentación y erosión que corresponden a siete unidades litoestratigráficas (de las cuales en el Vestíbulo sólo hemos estudiado las cinco primeras) y a cinco discontinuidades estratigráficas que las separan (Fig. 3). En este trabajo nos referiremos únicamente a la primera etapa en cuyos sedimentos se recuperaron los coprolitos que nos ocupan.

Esta primera etapa de la secuencia de la entrada de la Cueva de Nerja (Nerja 1), corresponde a los sedimentos de la Unidad 1 situados en una horquilla cronológica comprendida entre los 24.000 y los 20.000 BP. Esta unidad (potencia $120 \mathrm{~cm}$ ) corresponde al inicio de la sedimentación en ambas salas, que en el Vestíbulo descansa sobre una potente corteza estalagmítica mientras que en la Mina no se ha llegado a su base. Se compone de tres niveles en ambas salas (Vestíbulo: NV 13, NV 12, NV 11; Mina: NM 19, NM 18, NM 17) formados por arenas anaranjado-rojizas, con cantos autóctonos, que presentan idénticas características texturales y una cementación carbonatada, total o parcial, que les confiere cierta consistencia. Corresponde a una sedimentación en la que predominan los procesos de arroyada difusa por acción del agua de escorrentía superficial, en régimen laminar, que ocasionalmente pudo circular de forma ligeramente canalizada. En el nivel basal de esta unidad en el Vestíbulo (NV 13) se han encontrado los coprolitos que aquí se presentan junto a escasos restos óseos de macromamíferos, algunos de ellos en conexión anatómica, lo que podría implicar una baja energía en la sedimentación. En un momento intermedio se produce un aporte de cantos y bloques autóctonos. Con posterioridad a la sedimentación, tiene lugar una cementación carbonatada parcial de los materiales detríticos.

Por comparación con otras secuencias del Mediterráneo podríamos correlacionar esta etapa (Nerja 1) con los momentos finales de la fase Malladetes $\mathrm{C}$, en las últimas etapas de la penúltima oscilación fría del Würm reciente (Fumanal, 1986) que correspondería con el máximo glacial del estadio isotópico 2 (Shackleton y Opdyke, 1973). Este registro corresponde a una ocupación humana del Paleolítico superior inicial, cuya intensidad aumenta hacia el techo, detectándose en la parte basal coprolitos de grandes carnívoros que indican una ausencia del hombre en la cavidad en determinados momentos. El techo de esta unidad se encuentra erosionado existiendo un hiato entre esta y la Unidad 2.

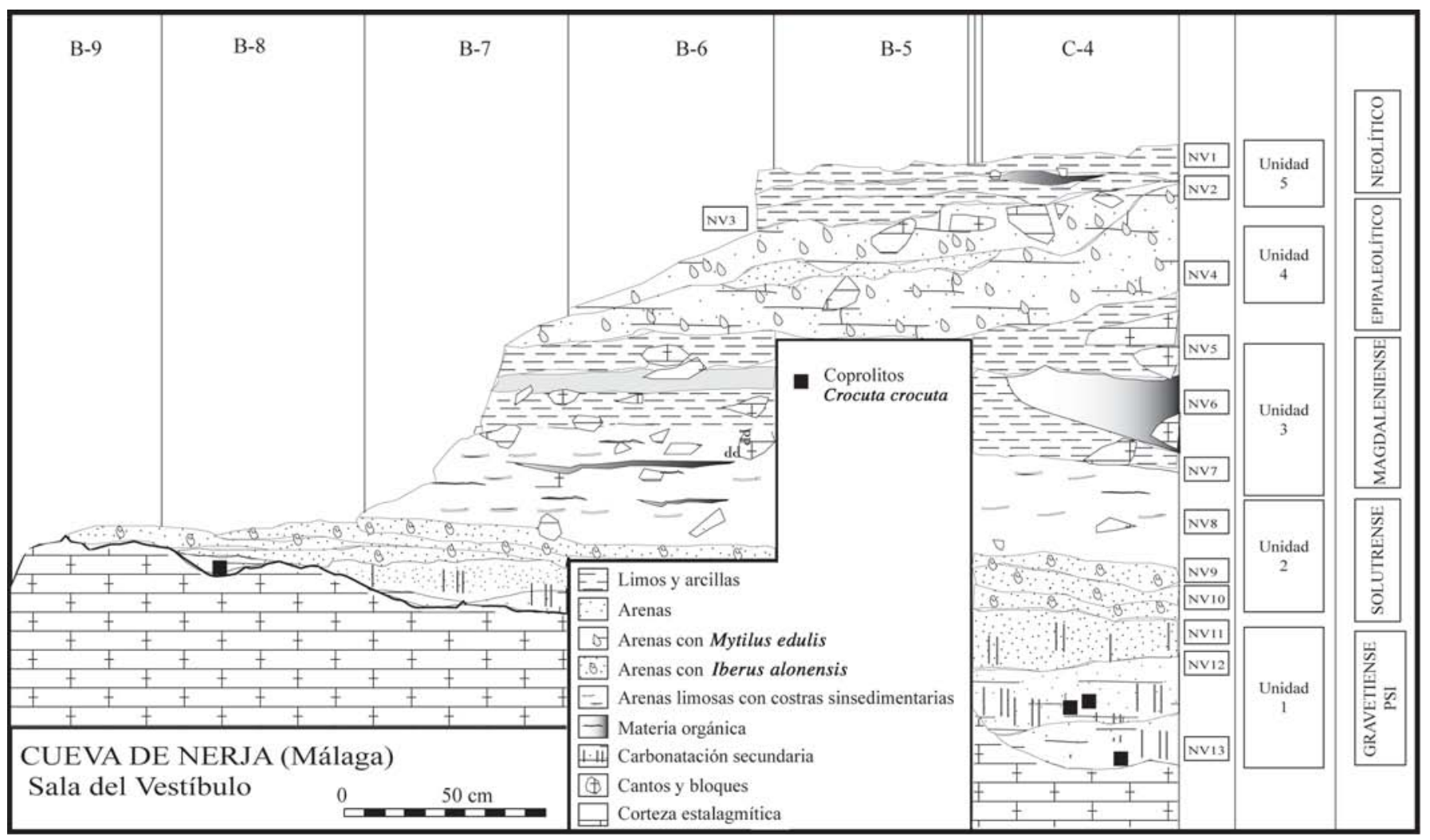

Figura 3. Secuencia estratigráfica de los depósitos de la Sala del vestíbulo de la Cueva de Nerja. Stratigraphic section of the deposits of Vestibulo Hall of Nerja's Cave. 


\section{CONTEXTO ARQUEOLÓGICO}

Desde el punto de vista arqueológico, conviene precisar que la excavación de los niveles correspondientes a la primera etapa de sedimentación se realizó en las dos salas mencionadas sobre una superficie limitada y desigual. En la Sala de la Mina se excavaron parcialmente 4 cuadrículas (D-5, E-5, F-5, E-4 y F-4), aunque sólo se alcanzó la corteza estalagmítica basal y, por tanto, se llevó a cabo la excavación completa de los tres niveles que componen esta etapa (NM 19, NM 18 y NM 17), en el cuadro E-5 y parte del D-5. Por su parte, en la Sala del Vestíbulo se realizó un sondeo de $1 \mathrm{~m}^{2}$ (cuadrícula C-4, Fig. 4) y, posteriormente, se procedió a la excavación de diferentes banquetas-testigo, originadas durante las excavaciones realizadas en esta sala en 1962-63. Esta intervención supuso la excavación parcial de los tramos medios y basales de la Unidad 1 en las cuadrículas A-8, B-6, B-7, B-8, B-9, C-7 y C-8 (Fig. 4).

Los coprolitos aquí descritos proceden de esta sala y más concretamente de las cuadrículas B-8 (1 ejemplar) y C-4 (3 ejemplares). Sobre muestras obtenidas de los procedentes de esta última cuadrícula (nivel 13-A) (Fig. 3), uno de nosotros (J.S.C.) realizó un análisis palinológico que dio resultados negativos. Su presencia en estos momentos de la secuencia plantea una interesante discusión que se puede resumir en dos alternativas.

A) La primera pasa por considerar un episodio inicial de ocupación de la cavidad por parte de hiénidos, que serían los agentes responsables de la acumulación de los coprolitos y de algunos restos de fauna que se describen a continuación. Los procesos posteriores de sedimentación de baja energía, acumularían una gran parte de este depósito en las cotas más bajas del Vestíbulo, concretamente en la cuadrícula C-4. La baja energía de este transporte se puede constatar además por la recuperación de una extremidad de Cervus elaphus en posición anatómica en C-4.

B) La segunda contemplaría varios episodios de ocupación de hiénidos, entre los que se intercalaría una primera presencia humana, registrada tan sólo en la cuadrícula C-4 y una última presencia de hiénidos registrada en la cuadrícula B-8.

En las figuras 3 y 5 se puede apreciar el importante buzamiento que ofrece el espeleotema basal del Vestíbulo: prácticamente 1 metro en el transecto horizontal de 5 $\mathrm{m}$ que media entre las cuadrículas C-4 y C-9. Esta paleotopografía debió influir en la posición y disposición de los niveles basales de esta sala y su discusión, en términos arqueológicos, remite a las dos alternativas expuestas más arriba.
Se dispone de dos dataciones radiocarbónicas para los depósitos del último de los levantamientos del nivel NV 13 , obtenidas a partir de muestras procedentes de la banqueta descrita anteriormente. En un caso se obtuvo mediante $\mathrm{C}^{14}$ convencional sobre un agregado de carbones dispersos de las cuadrículas B-6, B-7 y B-8 y su resultado es $24.300 \pm 1.400 \mathrm{BP}(\mathrm{UBAR}-340)$. La restante fue obtenida sobre un único resto de la cuadrícula B-7, identificado por E. Badal como Pinus pinea; el resultado obtenido mediante AMS es $24.480 \pm 110$ (Beta-131576). No disponemos de dataciones para la unidad basal de la cuadrícula C-4.

El último levantamiento de NV 13 de la banqueta-testigo (Fig. 5) supuso la excavación de $0,4 \mathrm{~m}^{3}$ de depósitos, mientras que la excavación de la totalidad de NV 13 en C-4, en sucesivos levantamientos arqueológicos (Fig. 4), totaliza aproximadamente $0,15 \mathrm{~m}^{3}$. Los materiales arqueológicos englobados en todas estas unidades de excavación conforman una muestra reducida, pero posiblemente significativa, de la primera presencia humana en esta sala de la cavidad.

De muro a techo, las cotas más bajas de los sedimentos excavados se sitúan en C-4. Se trata de una especie de cubeta trazada por la propia corteza estalagmítica en la que se recuperaron algunos restos de Bos sp. y Equus sp., muy concreccionados y pertenencientes a sendos individuos juveniles. Los restos están prácticamente enteros y presentan alguna marca ocasionada por mordedura de animales. A este paquete se asocia uno de los coprolitos aquí descritos, localizado a una cota de $-310 \mathrm{~cm}$ respecto del nivel 0 , y sin que dispongamos por ahora de ninguna evidencia cierta de la presencia humana en estos momentos (Fig. 4, 13-B).

Siguiendo el orden ascedente que marcan las cotas de profundidad, dos coprolitos más fueron recuperados en este cuadro asociados a una extremidad de Cervus elaphus, con abundantes concreciones y dispuesta en posición anatómica. Similares características presentaban algunos restos de un individuo juvenil de Equus sp. (mandíbula, pelvis y fémur) y que muy posiblemente pertenecieron al mismo ejemplar descrito en el párrafo anterior. Los dos coprolitos asociados a este paquete se situaban, aproximadamente, a $-305 \mathrm{~cm}$ respecto del nivel 0 (Fig. 4, 13-A). En esta unidad de excavación se recuperaron las primeras evidencias de la presencia humana si nos atenemos a sus cotas de profundidad: un raspador, una hojita con retoque alterno y un fragmento de varilla de asta, elementos de adorno, restos muy escasos de carbón y de fauna con fracturas de origen antrópico y, en algunos casos, alteraciones por exposición al fuego.

Por último, en la banqueta-testigo se recuperó el último de los coprolitos, en la cuadrícula B-8 y a una cota de profundidad de $-232 \mathrm{~cm}$ respecto del punto 0 (Fig. 5). Las evidencias directamente ligadas a la presencia humana son aquí igualmente abundantes, destacando la identificación 

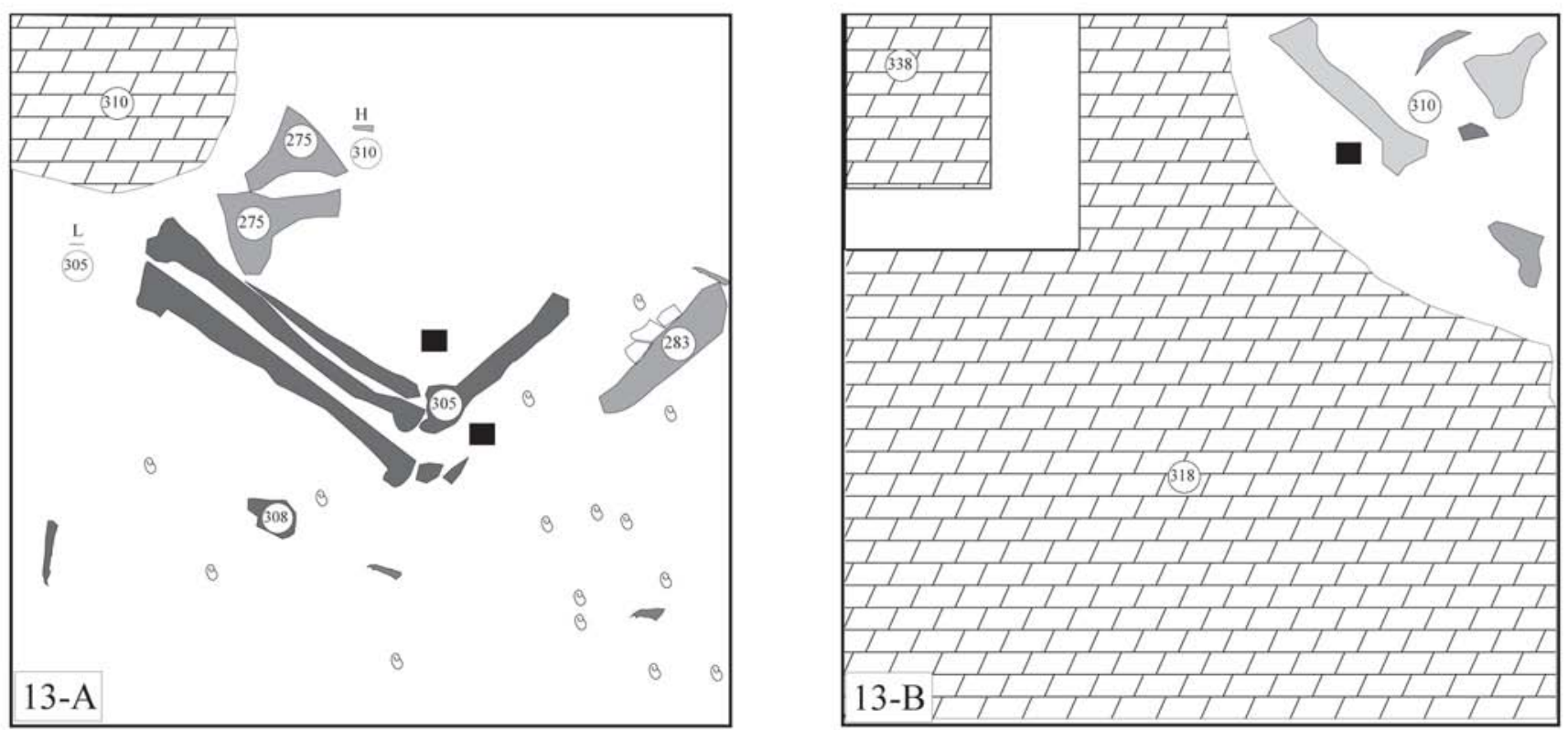

\begin{tabular}{|l|l|l|l|}
\hline \multicolumn{7}{|c|}{ Cueva de Nerja - Sala del Vestíbulo (1983-1984). Cuadrícula C-4, capa 13 } \\
\hline Coprolito de Crocuta crocuta spelaea & Equus caballus \\
\hline Fauna & Cervus elaphus & Bos primigenius \\
\hline S. & Iberus alonensis & Cotas de profundidad \\
\hline
\end{tabular}

\begin{tabular}{|l|l|}
\hline \multicolumn{2}{|c|}{ APORTES ANTRÓPICOS } \\
\hline Capa 13-A & Capa 13-B \\
\hline - 8 objetos líticos & \\
-1 frag. varilla de asta & \\
-1 Littorina sp. & \\
-1 colgante & Sin evidencias \\
- carbón Pinus sp. & \\
- restos fracturados y & \\
quemados de & \\
Cervus elaphus & \\
y Capra pyrenaica & \\
\hline
\end{tabular}

\begin{tabular}{|c|}
\hline APORTES NO ANTRÓPICOS \\
\hline Capa 13 \\
\hline - 3 coprolitos de Crocuta crocuta spelaea \\
- Equus caballus (restos de 1 individuo juvenil) \\
- mandíbula con D1, D2 y D3 \\
- pelvis, fémur, tibia y ulna \\
(repartidos entre NV12, NV13-A y NV13-B) \\
- Bos primigenius (restos de 1 individuo juvenil) \\
- metatarso y escápula (NV13-B) \\
- Cervus elaphus: \\
- extremidad en posición anatómica \\
(NV13-A)
\end{tabular}

Figura 4. Planimetría de la capa NV 13 en la cuadrícula C-4 e interpretación. Planimetry of level NV 13 in C-4 and interpretation. 


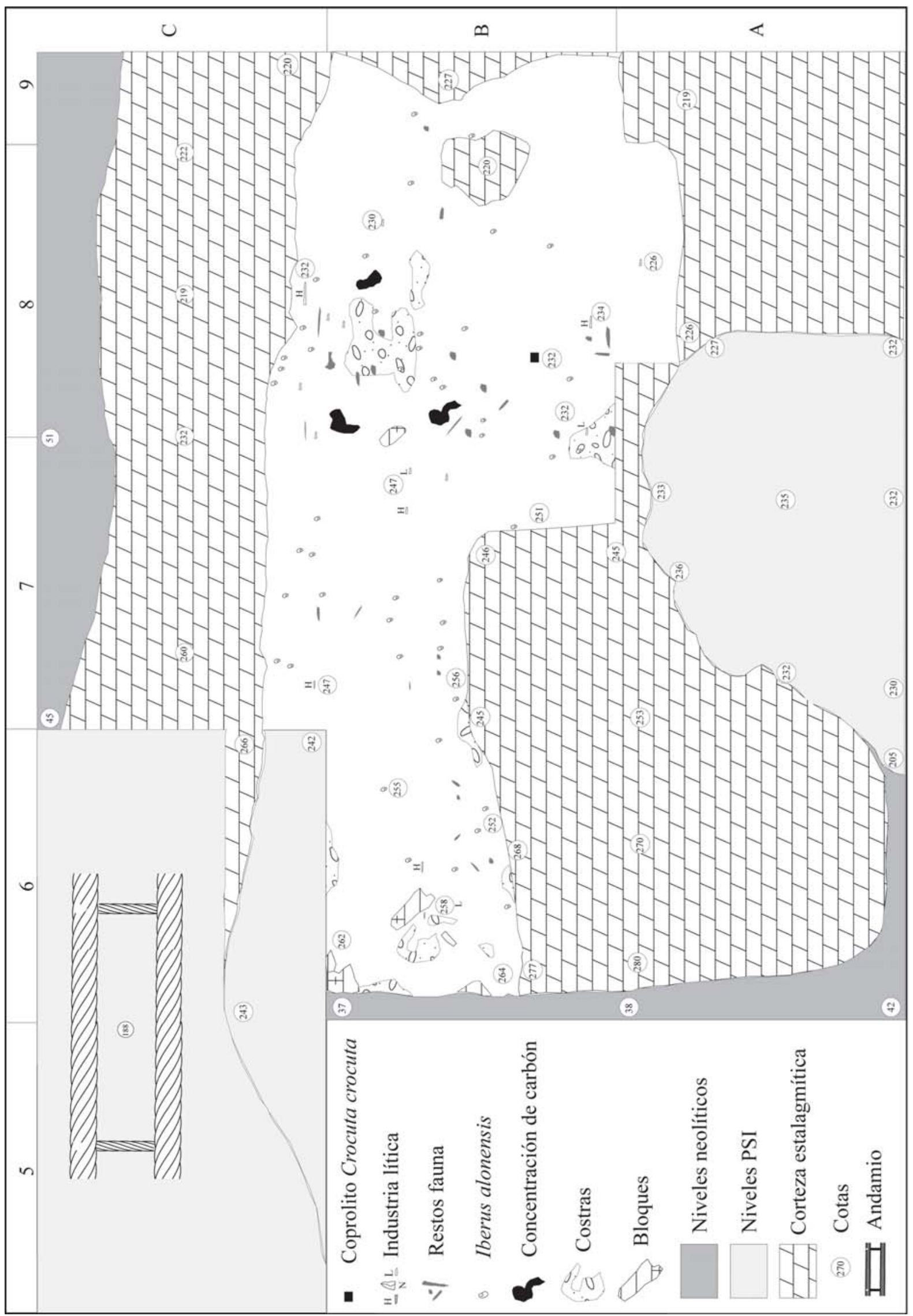

Figura 5. Dispersión de materiales en la base de la capa NV 13 de la Sala del Vestíbulo de la Cueva de Nerja. Materials dispersion from the base of level NV 13 (Vestíbulo Hall, Nerja's Cave). 
de tres concentraciones de carbón en la misma cuadrícula B-8, la recuperación de una punta de asta asi como de un reducido, pero significativo, conjunto de artefactos líticos, entre el que sobresalen raspadores, buriles, truncaduras, muescas y una punta de dorso acompañados de una interesante serie de soportes con retoques marginales y melladuras posiblemente originadas durante su utilización. La fauna procedente de la banqueta-testigo tiene un claro origen antrópico, deducido a partir de la morfología de las fracturas, alguna marca realizada con una herramienta lítica y las alteraciones por exposición al fuego.

En el listado de fauna que se adjunta (tabla 1) destaca la reducida presencia de restos de conejo, especie muy abundante en los catálogos del Paleolítico superior de la región mediterránea ibérica y cuyo origen antrópico es motivo de debate para estos momentos iniciales del $\mathrm{Pa}$ leolítico superior (Aura et al., 2002b).

La densidad de materiales claramente relacionables con la presencia humana es baja (entre 0 y 47 artefactos líticos retocados por metro cúbico de sedimentos, según cuadrículas), en comparación con los resultados obtenidos en capas superiores. No obstante, la tecnología y tipología del instrumental lítico y óseo recuperado en la banqueta testigo permiten su relación con la tecnología Gravetiense, adscripción que coincide con la edad obtenida en las dos dataciones antes mencionadas.

\begin{tabular}{lc}
\hline Nerja- Vestíbulo & N.V- 13 base \\
\hline Equus caballus & $7(5)$ \\
Bos primigenius & $2(2)$ \\
Cervus elaphus & $14(6)$ \\
Capra pyrenaica & 41 \\
Lynx pardina & 1 \\
Esquirlas & 85 \\
Quemados & 46 \\
Oryctolagus cuniculus & 76 \\
Aves & 10 \\
Reptiles & 1 \\
Quemados & 23 \\
\hline
\end{tabular}

Tabla 1. Restos de fauna manejados en este trabajo, entre paréntesis los restos relacionados con los aportes de hiénidos. No se incluyen los 4 coprolitos. Faunal remains used in this work (in brackets the fossils modified by hyaenids). Coprolites not included.

\section{IDENTIFICACIÓN Y DETERMINACIÓN DE LOS MATERIALES}

De los cuatro elementos recuperados en el nivel NV 13 de la Sala del Vestíbulo de la Cueva de Nerja, tres (C-
4; Fig. 6) son muy fragmentarios, mientras que el cuarto (B-8) se ha conservado completo pudiendo ser identificado como un lóbulo inferior de coprolito.

Los coprolitos de los grandes carnívoros trituradores de hueso constan generalmente de tres lóbulos cuando el ejemplar está completo (Arribas, 1994): uno superior, que suele ser el más grande, de forma cilíndrica (altura mayor o igual que el diámetro) con la cara superior convexa con un agudo apéndice y con la cara inferior cóncava; un lóbulo medio también cilíndrico, con la altura menor que el diámetro y las caras superior e inferior cóncavas; y un tercer lóbulo, el inferior, con forma de semicircunferencia, el diámetro mayor que la altura, la cara superior cóncava y la inferior convexa sin irregularidades significativas. Este último lóbulo es el primero en tocar el suelo tras la defecación.

El lóbulo en cuestión de la Cueva de Nerja tiene una altura estimada de $3 \mathrm{~cm}$, un diámetro de $4,8 \mathrm{~cm}$ y presenta su cara inferior convexa y lisa mientras que la cara superior tiene la depresión central de anclaje (con el lóbulo medio) característica de este tipo de elementos. El icnofósil está constituido por una matriz fosfática pulverulenta, homogénea y porosa, cementada por carbonatos, que engloba a algunos fragmentos centimétricos de hueso esponjoso no digeridos.

En tanto en cuanto que los coprolitos son icnofósiles, esto es, evidencias indirectas de la actividad de los organismos del pasado, y por tanto se ha de utilizar con ellos la nomenclatura parataxonómica, sí se puede conocer a que familia de carnívoros perteneció el organismo generador de tales estructuras. Las características enumeradas anteriormente para el icnofósil de la Cueva de Nerja permiten asegurar, por su tamaño, su estructura y por la presencia de fragmentos de hueso esponjoso de tejidos de macromamíferos, que el agente generador perteneció a la familia Hyaenidae. Los representantes de esta familia procesan habitualmente gran cantidad de huesos de macromamíferos con diversos objetivos alimenticios (Arribas, 1995), generando excrementos compuestos en más del $90 \%$ por los fosfatos no asimilados por el organismo.

Los cánidos, especialmente los zorros debido su dieta menos carnívora y más oportunista, también producen habitualmente excrementos susceptibles de fosilizar por sus altos contenidos en fases minerales. Ahora bien, existen tres diferencias esenciales entre los coprolitos de los zorros (cánidos s.l.) y los de los hiénidos, a saber: los coprolitos de los cánidos tiene uno o dos lóbulos; sus dimensiones son netamente inferiores a las de los hiénidos (están en función de diámetro del tracto intestinal); y por último y más importante, sus coprolitos son más heterogéneos y porosos concentrando en su interior fragmentos de los restos óseos de los animales de los que se alimentan, esto es, generalmente de lagomorfos y roedores. Por ello, en el interior de los coprolitos de los zorros es imposible encontrar fragmentos óseos de macro- 


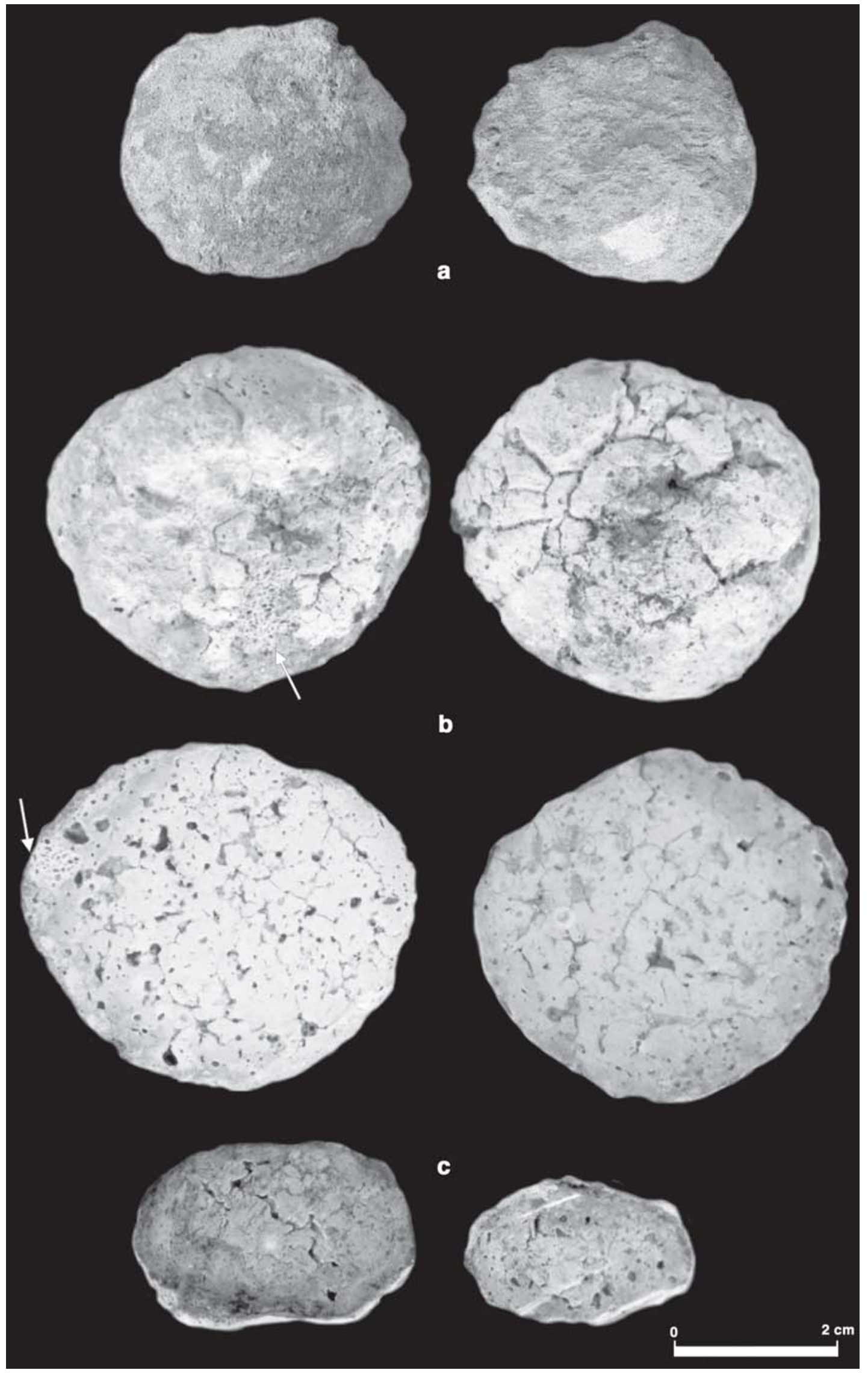

Figura 6. Coprolitos de la capa NV 13 (C-4): a, ejemplar entero; b y c, ejemplares cortados. Coprolites from level NV 13 (C-4): a, complete coprolite; $b$ and c, cutted samples. 
mamíferos ya que aunque puedan llegar a carroñear sus cadáveres no tienen la capacidad masticatoria de procesar sus huesos, mientras que es norma encontrar fragmentos identificables de los huesos de estos micromamíferos e incluso dientes completos o hemimandíbulas, como en los yacimientos pleistocenos de Villacastín (Arribas, 1994) y la Cueva de los Torrejones (Arribas et al., 1997; Díez Fernández-Lomana et al., 1998).

Por otra parte, los coprolitos de los hiénidos son unos icnofósiles que presentan una alta probabilidad de superar la etapa fosildiagenética por sus altos contenidos iniciales en fases minerales del grupo del apatito, ahora bien, este tipo de elementos no suelen superar la etapa bioestratinómica por efecto del «pisoteo». En la reducida superficie excavada de estos depósitos se apreció una baja presencia de fósiles de macromamíferos y la ausencia de estructuras de habitación de carnívoros en NV 13. Esta base documental, parece indicar que la presencia de estos restos fecales sólo puede ser explicada por la visita ocasional de algún hiénido a esta zona de la cueva.

Los excrementos de los carnívoros son objetos acumulados habitualmente en los lugares de habitación de estos animales, espacios en los que la actividad biológica es extrema y permanente. Cuando el lugar de acumulación de los excrementos se encuentra relativamente alejado del área de ocupación o es restringido, como en el yacimiento de Villacastín, las defecaciones no sufren un intenso «pisoteo»y se conservan coprolitos enteros (3 lóbulos). Cuando los excrementos se acumulan en las zonas de ocupación, ya sean esta cubiles o comederos, en los que existe una intensa actividad biológica, los excrementos son disgregados y dispersados por el «pisoteo» de los propios carnívoros. Esto produce una gran destrucción de restos y una mayor dispersión de los elementos sobre el substrato, conservándose lóbulos aislados como ocurre en algunos yacimientos kársticos como en la Cueva de los Torrejones (Nivel Entrada-4; cubil de Crocuta crocuta spelaea (Goldfuss, 1832); Arribas et al., 1997) o en otros yacimientos en medio abierto como Venta Micena [comedero junto a cubiles de Pachycrocuta brevirostris (Aymard, 1856) en un margen lacustre; Arribas et al., 1996; Arribas y Palmqvist, 1998; Palmqvist y Arribas, 2001].

En cuanto a la adscripción taxonómica del organismo generador es reseñable que los coprolitos de los hiénidos no presentan caracteres específicos que permitan definir ni si quiera a qué género de hiénidos perteneció dicho animal [las síntesis sobre los hiénidos del Pleistoceno español se encuentran en Arribas y Palmqvist (2001, en prensa)]. Cuando se encontraban coprolitos de hiénido en yacimientos del Plioceno superior existían dos candidatos que pudieran haberlos generado: o representantes del desaparecido género Chasmaporthetes Hay, 1921 (hiénido cursorial) o representantes del, también desaparecido, género Pachycrocuta Kretzoi, 1938 [Pachycrocuta perrieri (Croizet y Jobert, 1828), hiénidos netamente carroñeros posiblemente vinculados con las actuales hienas pardas]. Recientemente, se ha añadido un nuevo género de hiénido al registro paleontológico del Plioceno superior de España. Se trata de la hiena parda actual, Hyaena (Parahyaena) brunnea (Thunberg, 1820), representada por un cráneo, mandíbulas y huesos del esqueleto postcraneal en el nuevo yacimiento granadino de Fonelas P-1 (Arribas et al., 2001). La presencia de dicho hiénido en este yacimiento es la única cita conocida de la especie en todo el Plio-pleistoceno de Eurasia, pues su distribución paleobiogeográfica era exclusivamente africana. Chasmaporthetes lunensis Del Campana, 1914 y Pachycrocuta perrieri desaparecen del registro paleontológico español al final del Plioceno y son sustituidos en el Pleistoceno inferior por los representantes de la especie Pachycrocuta brevirostris, el gran hiénido recolector de huesos, único representante de la familia presente en España durante dicha serie. Este género no traspasa el límite Pleistoceno inferior-medio y es sustituido, ya en el Pleistoceno medio, por la hiena manchada (Crocuta crocuta Erxleben, 1777) que presenta en el registro español dos variedades: Crocuta crocuta intermedia (De Serres, 1828) en el Pleistoceno medio y una forma de mayores dimensiones, Crocuta crocuta spelaea, en el Pleistoceno superior. Esta última variedad se pensaba que estaba presente en las paleomastocenosis de España desde hace aproximadamente 128.000 años hasta el Gravetiense, siendo el único representante de esta familia durante el Pleistoceno superior. Sin embargo, recientemente se han presentado registros de esta variedad en depósitos de la Cueva de las Ventanas (provincia de Granada) cifrados en cronologías próximas al límite Pleistoceno superior-Holoceno (Carrión et al., 2001). Por tanto, y desde la perspectiva biocronológica, en el caso de los coprolitos de la Cueva de Nerja sólo hay un posible agente generador, la «hiena manchada de las cavernas» (Crocuta crocuta spelaea). Es evidente que esta determinación taxonómica es indirecta pero se ajusta a los conocimientos actuales que se poseen sobre la distribución bioestratigráfica de los hiénidos cuaternarios en España.

Con respecto a este tema conviene destacar una última cuestión. En la década de 1990 ha sido publicada la presencia del género Hyaena Brunnich, 1771, Hyaena spelaea nomen nudum (taxón no descrito formalmente ya que la especie o variedad spelaea pertenece al género Crocuta), en el yacimiento barcelonés del Abric Romaní (Aïmene et al., 1996). Los autores citan la coexistencia en dicha localidad de dos hiénidos en el Pleistoceno superior, los géneros Hyaena y Crocuta, de acuerdo con un trabajo paleontológico previo (Sánchez, 1989). En la asociación faunística descrita por dicha autora en la década de 1980 no está presente el género Hyaena, ya que todos los fósiles de hiénido por ella estudiados son determinados como Crocuta crocuta. Por otra parte, es conocido el registro de representantes del género Hyaena en yacimien- 
tos del Pleistoceno medio (Lunel Viel en Francia; Bonifay, 1971) y del Pleistoceno superior (Furninha en Portugal; Cardoso, 1993 y Genista cave en Gibraltar, Gran Bretaña; Kurten, 1968) en países de nuestro entorno geográfico. Dichos especimenes han sido determinados como Hyaena hyaena prisca De Serres, 1828, variedad pleistocena de gran tamaño de la actual hiena rayada (el más pequeño de los hiénidos modernos), de la que se desconocen sus ancestros en Europa. Sin duda, los materiales del límite Neógeno-Cuaternario de Fonelas P-1 aportarán luz sobre la filiación y significado de estas aisladas poblaciones pleistocenas pertenecientes al género Hyaena. Finalmente, es necesario recalcar que por el momento no se han encontrado fósiles asignables al género Hyaena en el Pleistoceno español, género fácilmente distinguible de la abundantemente registrada Crocuta, que por otra parte tiene su única aparición en el registro paleontológico de España hace aproximadamente 1,8 millones de años (Arribas et al., 2003).

\section{CONCLUSIONES}

La excavación de la sala del Vestíbulo ha proporcionado cerca de 20.000 restos de mamíferos, de los que algo más del $75 \%$ ha podido ser determinado a nivel de especie; y de igual forma, la sala de la Mina ha proporcionado un elevado número de restos. Los trabajos de Pérez Ripoll (1986, 1987) y Pérez Ripoll y Raga (1998) para la salas de la Mina y del Vestíbulo y de Morales y Martín (1995) para las de la Mina y de la Torca ofrecen un listado formado por 36 taxones entre los que cabe destacar la presencia de restos de Homo sapiens sapiens Linneo, 1758 a lo largo de la práctica totalidad de la secuencia del yacimiento, tanto en los niveles pleistocenos como en los correspondientes al Holoceno (García y Jiménez, 1995; Aura et al., 1998). La determinación taxonómica indirecta de Crocuta crocuta spelaea (Goldfuss, 1832) permite ampliar el registro paleomastológico de la Cueva de Nerja en una nueva especie, a la vez que plantea nuevos retos de cara al estudio de las ocupaciones de la entrada de la Cueva de Nerja en los primeros momentos de la secuencia sedimentaria, en donde hienas y hombres tuvieron una cierta competencia que finalmente se resolvió a favor de los segundos.

A la vista de todo lo anteriormente expuesto y teniendo en cuenta que tanto los sedimentos excavados en C-4 como en la banqueta-testigo forman parte de una misma unidad sedimentaria, NV 13, existe una dificultad a la hora de individualizar en su interior los diferentes aportes y ocupaciones humanas. A esta dificultad, intrínseca a buena parte de la documentación arqueológica de esta edad obtenida en cuevas, se añade la extensión excavada y su propia fragmentación. Teniendo en cuenta ambas variables y los datos manejados, resulta dificil decantarse por algu- na de las dos hipótesis alternativas expuestas anteriormente.

La primera nos situaría ante un escenario inicial en el que la cavidad es ocupada exclusivamente por hiénidos. Los aportes reconocibles se concentrarían en gran medida en C-4, ya que en la banqueta-testigo tan sólo se ha recuperado un coprolito pero no restos de fauna relacionables con su presencia. La paleotopografía de la corteza estalagmítica podría ser utilizada para explicar esta desigual distribución de restos sobre la superficie hasta ahora excavada.

La segunda de las alternativas abre diversos interrogantes que afectan tanto a la propia edad de los últimos hiénidos documentados en el sur de Iberia como al horizonte arqueológico y temporal de la primera presencia humana en esta sala, documentada en la cuadrícula C4. Se puede describir la unidad que hemos denominado para C-4 como NV 13-A como un palimpsesto en el que se acumulan coprolitos de hiénido y restos de fauna vinculables a su presencia junto con evidencias claras de la presencia humana (Fig. 4, NV 13-A). Su acumulación conjunta podría ser resultado de una redeposición de materiales anteriormente aportados o consecuencia de ocupaciones, alternativas y cortas, de hiénidos y humanos.

Es evidente que existen procesos postdeposicionales cuya acción genera situaciones arqueológicas de dificil evaluación. En el caso de la cuadrícula C-4 existen indicadores que apuntan en la dirección de considerar estas primeras evidencias de la presencia humana como parte de la ocupación claramente antrópica descrita para la banqueta-testigo. En este sentido, debemos mencionar que un artefacto fracturado recuperado en esta capa NV 13-A remonta con otro procedente de la capa NV 11, lo que vendría a relativizar el fuerte desajuste de cotas de profundidad observadas entre esta cuadrícula y la banquetatestigo. En todo caso, la evaluación correcta definitiva de este primer episodio de ocupación de la cavidad quizás se encuentre en obtener dataciones radiométricas sobre sendas muestras del escaso carbón recuperado en la capa NV 13-A de C-4 y sobre alguno de los restos de fauna que pueden ser vinculados a la acción de los hiénidos.

\section{REFERENCIAS}

Aïmene, M., Cáceres, I., Huguet, R., Ibañez, N., Rosell, J. y Saladié, P. 1996. Procesos de aprovechamiento de la fauna de Abric Romaní (Capellades, Barcelona). Actas de la II Reunión de Tafonomía y Fosilización, Zaragoza, 19-26.

Arribas, A. 1994. Los macromamíferos del yacimiento mesopleistoceno de Villacastín (Segovia, España). Boletín Geológico y Minero, 105, 344-361.

Arribas, A. 1995. Consideraciones cronológicas, tafonómicas y paleoecológicas del yacimiento cuaternario de Villa- 
castín (Segovia, España). Boletín Geológico y Minero, 106, 3-22.

Arribas, A. and Palmqvist, P. 1998. Taphonomy and paleoecology of an assemblage of large mammals: hyaenid activity in the Lower Pleistocene site at Venta Micena (Orce, Guadix-Baza Basin, Granada, Spain). Geobios, 31, 3-47.

Arribas, A. and Palmqvist, P. 2001. Hyaenids as collecting agents of bones in the Plio-Pleistocene record of Spain. Publicaciones del Seminario de Paleontología de Zaragoza, 5, 211-218.

Arribas, A. and Palmqvist, P. In press. Hyenids as a bone collecting agency in the Pleistocene fossil record of Spain: an overview of the taphonomy of Venta Micena and remarks on the paleobiology of the giant hyaena Pachycrocuta brevirostris. In: Hyenids In The CircumMediterranean: A Taphonomic Perspective (Eds. L.K. Horwitz and Ph. Fosse). Prehistoire et Anthropologie Méditerranéennes, U.M.R. 6636 et A.P.P.A.M., Aix-enProvence.

Arribas Herrera, A., Díez Fernández-Lomana, J.C. y Jordá Pardo, F.J. 1997. Primeras ocupaciones en los depósitos pleistocenos de la Cueva de los Torrejones (Sistema Central español, Tamajón, Guadalajara): litoestratigrafía y actividad biológica. Cuaternario y Geomorfología, 11, 55-66.

Arribas, A., Garrido, G. y Palmqvist, P. 2003. Primera cita de Hyaena (Parahyaena) brunnea (Thunberg, 1820) (Mammalia, Carnívora) fuera de África: el registro del yacimiento del Plioceno superior de Fonelas P-1 (Cuenca de Guadix-Baza, Granada, España). Geotemas, 5, 37-39.

Arribas, A., Palmqvist, P. y Martínez-Navarro, B. 1996. Estudio tafonómico cuantitativo de la asociación de macromamíferos de Venta Micena. Actas de la II Reunión de Tafonomía y Fosilización, Zaragoza, 27-38.

Arribas, A., Riquelme, J.A., Palmqvist, P., Garrido, G., Hernández, R., Laplana, C., Soria, J., Viseras, C., Durán, J.J., Gumiel, P., Robles, F., López-Martínez, J. y Carrión, J. 2001. Un nuevo yacimiento de grandes mamíferos villafranquienses en la Cuenca de Guadix (Granada): Fonelas P-1, primer registro de una fauna próxima al límite Plio-Pleistoceno en la Península Ibérica. Boletín Geológico y Minero, 112, 3-34.

Aura Tortosa, J.E., Jordá Pardo, J.F., González-Tablas, J., Bécares Pérez, J. y Sanchidrián Torti, J. 1998. Secuencia Arqueológica de la Cueva de Nerja: la Sala del Vestíbulo. In: Las Culturas del Pleistoceno Superior en Andalucía (Eds. J.L. Sanchidrián y M.D. Simón). Patronato de la Cueva de Nerja, Málaga, 217-236.

Aura Tortosa, J.E., Jordá Pardo, J.F., Pérez Ripol, M. y Rodrigo García, M.J. 2000. Sobre dunas, playas y calas. Los pescadores prehistóricos de la Cueva de Nerja (Málaga) y su expresión arqueológica en el tránsito Pleistoceno - Holoceno. Archivo de Prehistoria Levantina, 24, 9-39.

Aura Tortosa, J.E., Jordá Pardo, J.F., Pérez Ripol, M., Rodrigo García, M.J., Badal García, E. and Guillém
Calatayud, P. 2002a. The far south: the PleistoceneHolocene transitión in Nerja Cave (Andalucía, Spain). Quaternary International, 93-94, 19-30.

Aura, J.E., Villaverde, V., Pérez Ripoll. M., Martínez, R. and Guillem, P. 2002b. Big Game and Small Prey: Paleolithic and Epipaleolithic Economy from Valencia (Spain). Journal of Archaeological Method and Theory, 9, 209-262.

Badal, E. 1990. Aportaciones de la antracología al estudio del paisaje vegetal y su evolución en el cuaternario reciente en la costa mediterránea del País Valenciano y Andalucía (18.000-3.000 BP). Tesis Doctoral. Universitat de València (Inédita).

Badal, E. 1991. La vegetación durante el Paleolítico Superior en el País Valenciano y Andalucía. Resultados antracológicos. In: Arqueología Medioambiental a través de los macrorrestos vegetales (Ed. P. López). C.S.I.C. y Ayuntamiento de Madrid, Madrid, $21 \mathrm{pp}$.

Badal, E. 1996. La vegetation du Paleolithique Superieur et de l'Epipaleolithique aux alentours de la Cueva de Nerja (Málaga, Espagne). Actes du colloque de Périgueux 1995, Supplément à la Revue d'Archéométrie, 171-176.

Badal, E. 1998. El interés económico del pino piñonero para los habitantes de la Cueva de Nerja. In: Las Culturas del Pleistoceno Superior en Andalucía (Eds. J.L. Sanchidrián y M.D. Simón). Patronato de la Cueva de Nerja, Málaga, 287-300.

Boessneck, J. und von der Driesch, A. 1980. Tierknochenfunde aus vier Südspanischen Höhlen. Studien über frü Tierknochenfunde von der Iberischen Halbinsel, 7, 1-83.

Bonifay, M.F. 1971. Carnivores quaternaires du Sud-Est de la France. Memoires du Museum National d'Histoire Naturelle, N.S., serie C, 21, 43-274.

Cardoso, J. 1993. Contribuição para o conhecimento dos grandes mamíferos do Pleistocénico Superior de Portugal. Câmara Municipal de Oeiras, Oeiras, 567 pp..

Carrión, J.S., Riquelme, J.A., Navarro, C. and Munuera, M. 2001. Pollen in hyena coprolites reflects late glacial landscape in southern Spain. Palaeogeography, Palaeoclimatology and Palaeoecology, 176, 193-205.

Díez Fernández-Lomana, J.C., Jordá Pardo, J.F. and Arribas Herrera, A. 1998. Torrejones (Tamajón, Guadalajara, Spain). A hyaena den on human occupation. In: Économie Préhistorique. Les comportaments de subsistance au Paléolithique (Eds. J.P. Brugal, L. Meignen et M. Patou-Mathis). C.N.R.S. - Edition APDCA, Sophia Antipolis, 63-72.

Eastham, A. 1986. The birds of the Cueva de Nerja. In: $L a$ Prehistoria de la Cueva de Nerja (Ed. J.F. Jordá Pardo). Trabajos sobre la Cueva de Nerja, 1, Patronato de la Cueva de Nerja, Málaga, 107-131.

Fumanal, M.P. 1986. Sedimentología y clima en el País valenciano. Las cuevas habitadas en el Cuaternario reciente. Servicio de Investigación Prehistórica, Trabajos Varios, 83, Valencia, 207 pp.

García Sánchez, M. y Jiménez Brobeil, S.A. 1995. Los restos humanos de la Cueva de Nerja (Málaga): estudio antropológico. In: La Prehistoria de la Cueva de Nerja (Ed. J.F. Jordá Pardo). Trabajos sobre la Cueva de Nerja, 1, Patronato de la Cueva de Nerja, Málaga, 403-423. 
Guillem Calatayud, P. 1997. Estudio preliminar de los micromamíferos (Rodentia, Insectivora y Chiroptera) de la Cueva de Nerja, Sala del Vestíbulo, 15 pp. (Inédito).

Hernández Carrasquilla, F. 1995. Cueva de Nerja: las Aves de las campañas de 1980 y 1982. In: Fauna de la Cueva de Nerja I, (Eds. M. Pellicer, M. y A. Morales). Trabajos sobre la Cueva de Nerja, 5, Patronato de la Cueva de Nerja, Málaga, 219-293.

Jiménez Fuentes, E. 1986. Los quelonios de la Cueva de Nerja. In: La Prehistoria de la Cueva de Nerja (Ed. J.F. Jordá Pardo). Trabajos sobre la Cueva de Nerja, 1, Patronato de la Cueva de Nerja, Málaga, 133-143.

Jordá Pardo, J.F. 1981. La malacofauna de la Cueva de Nerja (I). Zephyrvs, 32-33, 87-99.

Jordá Pardo, J.F. 1982. La malacofauna de la Cueva de Nerja (II): Los elementos ornamentales. Zephyrvs, 34-35, 89-98.

Jordá Pardo, J.F. 1983. La secuencia malacológica de la Cueva de Nerja (Málaga). Excavaciones de 1982. Cuadernos do Laboratorio Xeolóxico de Laxe, 5, 55-71.

Jordá Pardo, J.F. 1985. La malacofauna de la Cueva de Nerja (III): Evolución medioambiental y técnicas de marisqueo. Zephyrvs, 37-38, 143-154.

Jordá Pardo, J.F. 1986a. Estratigrafía y Sedimentología de la Cueva de Nerja (Salas de la Mina y del Vestíbulo). In: La Prehistoria de la Cueva de Nerja (Ed. J.F. Jordá Pardo). Trabajos sobre la Cueva de Nerja, 1, Patronato de la Cueva de Nerja, Málaga, 39-97.

Jordá Pardo, J.F. 1986b. La fauna malacológica de la Cueva de Nerja. In: La Prehistoria de la Cueva de Nerja. (Ed. J.F. Jordá Pardo). Trabajos sobre la Cueva de Nerja, 1, Patronato de la Cueva de Nerja, Málaga, 145-177.

Jordá Pardo, J.F. 1992. Neógeno y Cuaternario del extremo oriental de la costa de Málaga. Tesis Doctoral. Universidad de Salamanca, 888 pp. (Inédita).

Jordá Pardo, J.F., Aura Tortosa, J.E. y Jordá Cerdá, F. 1990. El límite Pleistoceno - Holoceno en el yacimiento de la Cueva de Nerja (Málaga). Geogaceta, 8, 102-104.

Jordá Pardo, J.F., Aura Tortosa, J. E., Rodrigo García, M. J., Pérez Ripoll, M. y Badal García, E. 2003. El registro paleobiológico cuaternario del yacimiento arqueológico de la Cueva de Nerja (Málaga, España). Boletín de la Real Sociedad Española de Historia Natural (sección geológica), 98, 73-89.

Kurtén, B. 1968. Pleistocene Mammals of Europe. Weidenfeld and Nicolson, London, $317 \mathrm{pp}$..

Morales Muñíz, A. y Martín Díaz, J.M. 1995. Los mamíferos de la Cueva de Nerja: análisis de las cuadrículas NM80A, NM-80B y NT-82. In: Fauna de la Cueva de Nerja I (Eds. M. Pellicer, M. y A. Morales). Trabajos sobre la
Cueva de Nerja, 5, Patronato de la Cueva de Nerja, Málaga, 57-159.

Palmqvist, P. and Arribas, A. 2001. Taphonomic decoding of the paleobiological information locked in a lower Pleistocene assemblage of large mammals. Paleobiology, 27, 512-530.

Pellicer, M. y Acosta, P. 1997. Síntesis: El Neolítico y Calcolítico en Andalucía. In: El Neolítico y Calcolítico de la Cueva de Nerja (Eds. M. Pellicer y P. Acosta). Trabajos sobre la Cueva de Nerja, 6, Patronato de la Cueva de Nerja, Málaga, 373-393.

Pérez Ripoll, M. 1986. Avance al estudio de los mamíferos de la Cueva de Nerja. In: La Prehistoria de la Cueva de Nerja (Ed. J.F. Jordá Pardo). Trabajos sobre la Cueva de Nerja, 1, Patronato de la Cueva de Nerja, Málaga, 99-106.

Pérez Ripoll, M. 1997. Estudio zooarqueológico de la Cueva de Nerja: la Sala del Vestíbulo. 109 pp. (Inédito).

Pérez Ripoll, M y Raga, J.A. 1998. Los mamíferos marinos en la vida y en el arte de la Prehistoria de la Cueva de Nerja. In: Las Culturas del Pleistoceno Superior en Andalucía (Eds. J.L. Sanchidrián y M.D. Simón). Patronato de la Cueva de Nerja, Málaga, 251-275.

Rodrigo García, M.J. 1991. Remains of Melanogrammus aeglefinus (Linnaeus, 1758) in the Pleistocene-Holocene Passage of the Cave of Nerja (Málaga, Spain). In: Schriften aus der Archäeologist-Zoologishen Arbeistsgruppe Schleswig (Ed. W. van Neer). VI Meeting of the Fish Remains Working Group (ICAZ), Kiel, 348-351.

Roselló, E., Morales, A. y Cañas, J.M. 1995. Estudio ictioarqueológico de la Cueva de Nerja (Prov. De Málaga): resultados de las campañas de 1980 y 1982. In: Fauna de la Cueva de Nerja I (Eds. M. Pellicer y A. Morales). Trabajos sobre la Cueva de Nerja, 5, Patronato de la Cueva de Nerja, Málaga, 164-217.

Sánchez, B. 1989. La fauna de mamíferos del Pleistoceno superior del Abric Romaní (Capellades, Barcelona). Actas de las IV Jornadas de Paleontología, Salamanca, 331-347.

Serrano, F., Lozano Francisco, M.C., Vera Peláez, J.L. y Guerra Merchán, A. 1995. Malacofauna en yacimientos prehistóricos de la Cueva de Nerja. In: Fauna de la Cueva de Nerja I (Eds. M. Pellicer, M. y A. Morales). Trabajos sobre la Cueva de Nerja, 5, Patronato de la Cueva de Nerja, Málaga, 297-373.

Shackleton, N.J. and Opdyke, N.D. 1973. Oxygen isotope and paleomagnetic stratigraphy of equatorial Pacific core V28-238: oxigen isotope temperature and ice volumes on 10 (5) year and 10 (6) year scale. Quaternary Research, 3, 39-55. 